\title{
Self-rated walking pace and all-cause, cardiovascular disease and cancer mortality: individual participant pooled analysis of 50225 walkers from 11 population British cohorts
}

\author{
Emmanuel Stamatakis, ${ }^{1,2}$ Paul Kelly, ${ }^{3}$ Tessa Strain, ${ }^{3,4}$ Elaine M Murtagh, ${ }^{5}$ Ding Ding, ${ }^{1,2}$ \\ Marie H Murphy ${ }^{6}$
}

- Additional material is published online only. To view please visit the journal online (http://dx.doi.org/10.1136/ bjsports-2017-098677)

${ }^{1}$ Epidemiology Unit, Charles Perkins Centre, University of Sydney, Sydney, New South Wales, Australia

2Prevention Research Collaboration, Faculty of Medicine and Health, School of Public Health, University of Sydney, Sydney, New South Wales, Australia ${ }^{3}$ Physical Activity for Health Research Centre, Institute for Sport, Physical Education and Health Sciences, University of Edinburgh, Edinburgh, UK ${ }^{4}$ MRC Epidemiology Unit, University of Cambridge, Cambridge, UK

${ }^{5}$ Mary Immaculate College, University of Limerick, Limerick, Ireland

${ }^{6}$ Sport and Exercise Sciences Research Institute, University of Ulster, Coleraine, Northern Ireland

\section{Correspondence to}

Professor Emmanuel Stamatakis, Epidemiology Unit, Charles Perkins Centre, University of Sydney, Camperdown, NSW 2006, Australia;

emmanuel.stamatakis@sydney. edu.au

Received 11 October 2017 Revised 24 January 2018 Accepted 8 February 2018
Check for updates

To cite: Stamatakis E, Kelly $P_{\text {, }}$ Strain T, et al. Br J Sports Med 2018:52:761-768.

\begin{abstract}
Background/objectives Walking pace is associated with risk of premature mortality. However, whether this relationship is independent of total volume of physical activity and highest physical activity intensity remains unclear. We examined the associations between walking pace and cause-specific mortality, investigating the potential modifying effect of factors such as total physical activity volume, highest physical activity intensity, age, sex and body mass index (BMI).
\end{abstract}

Methods Prospective pooled analysis of 11 populationbased baseline surveys in England and Scotland between 1994 and2008 that were linked with mortality records. Multivariate-adjusted Cox proportional hazards models examined associations between walking pace (slow, average, brisk/fast) and all-cause, cancer and cardiovascular disease (CVD) mortality.

Results 50225 walkers were entered in the core analyses. Among participants who did not experience an event in the first 2 years of follow-up ( $n=49731$ ), walking at an average or brisk/fast pace was associated with a reduced risk of all-cause $(20 \%(95 \% \mathrm{Cl} 12 \%$ to $28 \%$ ) and $24 \%$ (95\% Cl $13 \%$ to $33 \%$ ), respectively) and CVD mortality (24\% (95\% CI 9\% to 36\%) and $21 \%(95 \% \mathrm{Cl} 1 \%$ to $38 \%)$, respectively), compared with reporting walking at a slow pace. In stratified analyses, such associations were evident among those over 50 years, those not meeting the physical activity recommendations and those who did not undertake vigorous-intensity activity. There were no interactions by sex or BMI. No associations were seen between pace and cancer mortality.

Conclusion Walking benefits health. Assuming causality, these analyses suggest that increasing walking pace could reduce risk for all-cause and CVD mortality. Walking pace could be emphasised in public health messages, especially in situations when increase in walking volume or frequency is less feasible.

\section{INTRODUCTION}

Increasing population level walking remains a key focus of physical activity (PA) promotion. Regular walking is known to confer many physical, mental and social health benefits. ${ }^{1}$ Meta-analyses of cohort studies have sought to quantify the association between regular walking and reduction in risk for all-cause mortality (ACM). ${ }^{2-4}$ Kelly et al estimated that after adjustment for other PA, walking at a volume equivalent to PA guidelines was associated with an $11 \%$ reduction in risk for ACM compared with no walking.

Considering specific health endpoints, cardiovascular disease (CVD) and cancer are the two most common avoidable causes of mortality in the UK. ${ }^{6}$ Hamer and Chida conducted a meta-analysis of 13 cohort studies and found a 31\% reduction in risk of CVD mortality in the highest walking categories compared with the lowest walking volume/intensity category. $^{2}$ A recent large analysis of over 250000 adults in the UK found walking to work was associated with a $36 \%$ reduction in risk of CVD mortality compared with non-active commuting. ${ }^{7}$ The results for cancer mortality are less clear, with, for example, Matthews et $a l^{8}$ and Celis-Morales et $a l^{7}$ finding no significant associations between walking volume and cancer mortality in large cohort studies. ${ }^{78}$

According to principles of overload, a higher relative activity intensity achieved by a faster pace of walking would provide the stimulus to produce a greater physiological response, and more substantial or even additional health benefits. Acute studies have shown that walking at a faster pace results in greater physiological responses. ${ }^{1}$ However, while total volume of walking, for example, by distance or time has been frequently studied, ${ }^{2-5}$ less is known about the long-term health effects of habitual walking pace.

A Copenhagen City Heart Study analysis ${ }^{9}$ reported reduced risk of heart failure for moderate and high walking speed compared with slow speed. The authors also suggested that walking pace may have a stronger association with heart failure than total duration of walking. Manson et $a^{10}$ found that among 73743 postmenopausal women aged 50-79 years, walking pace was associated with reduced incidence of CVD in a dose-response fashion. In a 40-year follow-up of the Whitehall study of 6981 British civil servants, Batty et al ${ }^{11}$ compared slow walking pace with high walking pace and found a reduced risk of all-cause, coronary heart disease and total cancer mortality. None of these studies adjusted for total volume of PA and it is therefore unclear if the reported effects were partly attributable to the higher overall activity levels of brisk/fast walkers.

A recent analysis of 420000 UK Biobank participants found significant associations between higher walking pace and reduced risk of all-cause and CVD mortality, but inconsistent findings for cancer 
mortality. ${ }^{12}$ However, the UK Biobank had a response rate of $5.5 \%$ and concerns have been raised about the generalisability of non-genetic associations from very unrepresentative cohorts. ${ }^{13}$

In summary, walking pace has been found to be associated with reduced risk of all-cause and cause-specific mortality in a number of cohort studies but the literature on the whole has not addressed independence from total PA robustly. There remains a knowledge gap about the independence of the relationships between walking pace and mortality outcomes in large population cohorts.

Our aim was to examine the associations between self-reported walking pace with all-cause, CVD and cancer mortality in a population representative sample of 11 pooled population British cohorts. A secondary aim was to better understand the role of total and total PA, sex, age and body mass index (BMI) as potential moderators of these associations.

\section{METHODS}

\section{Sample}

The Health Survey for England (HSE) ${ }^{14}$ and the Scottish Health Survey $(\mathrm{SHeS})^{15}$ are established household-based population surveillance studies running since 1991 and 1995, respectively. Each year, samples are selected using a multistage, stratified probability design aimed at recruiting a nationally representative sample of adults living in private households. Trained interviewers visited the selected households, and the recruited participants were administered the study questionnaires. 91.6\% of survey participants gave written consent to have their death flagged on the NHS Central Mortality Register. For this analysis, we used data from HSE 1994, 1997, 1998, 1999, 2003, 2004, 2006 and 2008 and SHeS 1995, 1998 and 2003. As population mortality rates increase evidently from the fourth decade of life, we included individuals aged $\geq 30$ years old who reported at least one occasion of walking in the last 4 weeks, had no doctor-diagnosed or self-reported (long-standing illness module) ischaemic heart disease, angina or stroke, and no prevalent cancer through cancer registration records or self-reported (long-standing illness module).

\section{Mortality outcomes}

Participants were followed up for mortality until 31 December 2009 (SHeS) or 31 March 2011 (HSE). Diagnoses for primary causes of death were recorded according to the International Classification of Diseases, Ninth Revision (ICD-9) and Tenth Revision (ICD-10). Cancer deaths were identified using ICD-9 140.0-239.9 and ICD-10 C00.0-D48.9 codes; CVD deaths were identified using ICD-9 390.0-459.9 and ICD-10 I01.0-I99 codes.

\section{Assessment of walking and other PA}

PA was assessed using an interviewer-administered questionnaire that inquired about walking, domestic PA and participation in sports and exercises in the 4 weeks prior to the interview. An occasion of walking was variously defined as at least $10 \mathrm{~min}$ or at least $15 \mathrm{~min}$ or at least $30 \mathrm{~min}$ in the different baseline surveys ${ }^{16}$ Walking was assessed using a question on number of days walked in the last 4 weeks, the average amount of time spent walking on each day and the usual walking pace ('which of the following describes your usual walking pace: slow pace, average pace, fairly brisk pace, fast pace-at least $4 \mathrm{mph}$ '). Because some baseline surveys (HSE 1994/1999/2003/2004; SHeS 1995) did not enquire about walking duration per reported occasion, we imputed this information based on the age and sex-specific estimates of HSE 1997/1998 (that included duration questions) using methods described elsewhere. ${ }^{16}$. All PA variables were summarised to reflect weekly averages for easier comparison with currently recommended amounts. The criterion validity of the walking-related questions is unknown. In a convergent validity study of over 2000 adults, the Spearman's correlation coefficients between accelerometry counts and walking of brisk/ fast pace were 0.35 (95\% CI 0.31 to 0.40 ) for women and 0.28 (95\% CI 0.23 to 0.34 ) for men. ${ }^{17}$ The equivalent coefficients for total weekly questionnaire derived metabolic equivalents (MET)-min were 0.41 (95\% CI 0.36 to 0.46 ) for women and 0.32 (95\% CI 0.26 to 0.38 ) for men. ${ }^{17}$

The PA compendium ${ }^{18}$ was used to assign the MET for all activities to calculate total MET-hours/week. We estimated adherence to the general guideline ${ }^{19}$ as accumulating weekly at least $150 \mathrm{~min}$ of moderate intensity or $75 \mathrm{~min}$ of vigorous intensity or equivalent combinations of moderate and vigorous PA. ${ }^{19}$ We also calculated the highest PA intensity reached on at least one occasion over the last 4 weeks that the PA questionnaire time frame covered (light/moderate/vigorous).

\section{Covariates}

Height and weight were measured by the interviewers using standard protocols ${ }^{14}{ }^{15}$; BMI was calculated as weight (in kilograms) divided by height (in metres) squared. Additional questions assessed age, educational attainment (age completed full-time education), presence of long-standing illness, weekly frequency of alcohol consumption, smoking habits (never smoker, ex-smoker, currently smoking 1-9 cigarettes/day, currently smoking 10-19/ day, currently smoking $\geq 20$ /day), psychological distress/depression (12-point General Health Questionnaire (GHQ) score), total (non-walking) leisure time PA volume (MET-hours/week) and total walking volume (MET-hours/week), and highest PA intensity reached on at least one occasion.

\section{Statistical analysis}

Analyses were conducted using SPSS V.22 (SPSS). Cox proportional hazards models with time in study as the time scale were used to examine the associations between walking pace and all-cause, CVD and cancer mortality with 'slow pace' as the reference category. Walking pace was originally entered in its original four-category format but the low number of events in the 'fast pace' category resulted in unstable estimates and broad 95\% CIs; for this reason, all main analyses were carried out with 'fairly brisk' and 'fast' pace categories collapsed into one group. In a supplemental analysis, we entered walking pace in its original format.

Kaplan-Meier log-minus-log plots were used to examine the proportional hazards assumption and no violations were observed. Analyses were adjusted for age, sex and all covariates listed above. Occupational PA could not be used in the calculation of PA volume because of its non-quantitative nature (it was reported as very/fairly/not very/not at all physically active). Also, we chose not to adjust for occupational PA level in the main Cox models because of the large number of missing values $(n \approx 27000)$ due to the corresponding question missing from SHeS 1995 and for responses being dependant on employment status.

We examined effect modification by sex, age and total PA level using type 3 Wald $\mathrm{X}^{2}$ statistics for the interaction term in the partially adjusted (for age, sex and cohort/year) model. For interactions with $\mathrm{p}<0.010$ we performed stratified analyses. ${ }^{19}$ To minimise the possibility of spurious associations due to occult 
Table 1 Baseline characteristics of the sample by walking pace. Walkers aged 30 years and over with no diagnosed cardiovascular disease or cancer at baseline

\begin{tabular}{|c|c|c|c|c|c|}
\hline & \multicolumn{4}{|l|}{ Walking pace } & \multirow[b]{2}{*}{ P value* } \\
\hline & Slow pace & Average pace & $\begin{array}{l}\text { Fairly brisk } \\
\text { pace }\end{array}$ & Fast pace & \\
\hline Age, mean (SD) (years) & 57.8 & 51.1 & 47.7 & 44.6 & $<0.001$ \\
\hline Sex (\% female) & 61.0 & 58.5 & 52.2 & 40.5 & $<0.001$ \\
\hline Body mass index, mean (SD) $\left(\mathrm{kg} / \mathrm{m}^{2}\right)$ & $28.6(5.7)$ & $27.3(4.7)$ & $26.1(4.1)$ & $25.5(3.9)$ & $<0.001$ \\
\hline Long-standing illnesst (\%) & 64.6 & 41.9 & 35.4 & 33.0 & $<0.001$ \\
\hline Smoking (\% current) & 23.9 & 24.0 & 21.5 & 27.5 & $<0.001$ \\
\hline Alcohol frequency ( $\% \geq 5$ times/week $) \ddagger$ & 18.7 & 18.7 & 22.4 & 24.5 & $<0.001$ \\
\hline Psychological distress ( $\%$ with General Health Questionnaire score $\geq 4) \S$ & 20.1 & 11.9 & 11.6 & 12.2 & $<0.001$ \\
\hline Age finished education ( $\%$ finished age $19+$ ) & 12.9 & 18.1 & 26.3 & 29.0 & $<0.001$ \\
\hline Meeting the physical activity recommendations & 8.9 & 17.2 & 47.5 & 52.4 & $<0.001$ \\
\hline \multicolumn{6}{|l|}{ Highest PA intensity reached (\%) } \\
\hline No physical activity & 11.7 & 7.9 & 4.9 & 4.9 & \multirow[t]{4}{*}{$<0.001$} \\
\hline Light intensity only & 63.4 & 52.5 & 14.4 & 14.8 & \\
\hline Reached moderate intensity & 11.8 & 15.3 & 42.9 & 37.2 & \\
\hline Reached vigorous intensity & 13.1 & 24.3 & 37.7 & 43.0 & \\
\hline MET-hours of physical activity per week, median (SE) & $8.0(0.38)$ & $17.0(0.20)$ & $23.1(0.31)$ & $32.0(0.80)$ & $<0.001$ \\
\hline Number of days walked per week, median (SE) & $2.0(0.04)$ & $2.5(0.02)$ & $2.5(0.02)$ & $3.0(0.05)$ & $<0.001$ \\
\hline MET-hours of walking per week (any pace), median (SE) & $2.3 .5(0.12)$ & $4.9(0.08)$ & $6.1(0.12)$ & $9.4(0.39)$ & $<0.001$ \\
\hline
\end{tabular}

The Health Survey for England and Scottish Health Survey $(n=50225)$.

* $P$ value calculated using Kruskal-Wallis test for continuous variables and likelihood ratio $\mathrm{X}^{2}$ test for categorical variables.

tDichotomous variable derived from responses to a series of questions (yes/no) on illness within eight listed body systems (eg, nervous system, digestive system, heart and circulatory system, and so on). At least one illness required to have long-standing illness.

$\ddagger$ Derived from the question, 'On how many days in the last 7 days did you have an alcoholic drink?'

$\S$ General Health Questionnaire comprises 12 questions related to psychological health (eg, concentration, feeling depressed, and so on); the categories were $0,1-3$ and $\geq 4$.

IAt least $150 \mathrm{~min}$ of moderate-intensity activity or $75 \mathrm{~min} /$ week of vigorous-intensity activity or equivalent combinations of moderate and vigorous activities.

MET, metabolic equivalent; PA, physical activity.

disease we ran a sensitivity analysis where we both included and excluded participants who died in the first 24 months of follow-up. This manuscript adheres to the Strengthening the Reporting of Observational Studies in Epidemiology standards for reporting of observational studies (STROBE).

\section{RESULTS}

In total, 65381 participants were initially considered; 4811 participants ( $8.4 \%$ of total eligible) did not consent to follow-up and were excluded. The variables with the highest number of missing data were BMI $(n=6346)$, GHQ score $(n=2444)$ and smoking $(n=151)$. In total, there were 3617 deaths from any cause including 1014 from CVD and 1276 from cancer causes. The mean follow-up was $9.2(\mathrm{SD}=4.6)$ years, corresponding to 469235 person-years. Table 1 presents the sample characteristics for the 50225 individuals in the core analytical sample. Slower walking pace was associated with older age, female sex, higher BMI scores, reporting a long-standing illness at baseline and psychological distress. Faster walking pace was associated with being a smoker, high frequency of alcohol consumption, finishing education after age 19 years, meeting the PA recommendations, participating in higher intensity PA, high volumes of total non-occupational PA, and higher frequency and total walking volume. Walking pace (in its original four-group format) showed low magnitude correlations with total leisure time PA volume (Spearman $r=0.25)$ and total walking volume $(r=0.20)$.

Table 2 presents the associations between walking pace and the three mortality outcomes with all participants who had an event in the first 24 months of the follow-up excluded ( $n=49731)$. In the fully adjusted models, walking at an average pace was associated with a risk reduction for ACM of $20 \%$ (95\% CI $12 \%$ to
$28 \%$ ) compared with those walking at a slow pace. The respective risk reduction for those walking at brisk/fast pace was $24 \%$ (13\%-33\%). For CVD mortality, walking at an average pace was associated with a $24 \%(9 \%-36 \%)$ risk reduction and walking at a brisk/fast pace was associated with 21\% (1\%-38\%) risk reduction compared with those walking at a slow pace. There was no evidence to suggest walking at an average or brisk/fast pace was associated with a significant risk reduction in cancer mortality $(\mathrm{HR}=1.08$ (0.89-1.31) and $\mathrm{HR}=1.02$ (0.81-1.29), respectively). The results were similar in direction and magnitude when those who had an event in the first 24 months were included (online supplementary table 1 ). When the walking pace variable was entered in its original four-group format (online supplementary table 2) associations were similar in magnitude and direction but likely due to lower number of events, the $95 \%$ CIs of the fast pace group were very wide and included one for all three outcomes. Repeating all above analyses with the models adjusted for total duration of moderate to vigorous PA (MVPA) and light-intensity activity (instead of average MET-hours/week) produced almost identical results, for example, the HR $(95 \% \mathrm{CI})$ for ACM in the average pace group changed from 0.80 (0.72 to $0.88)$ to $0.80(0.73$ to 0.88$)$; in the brisk/fast group it changed from $0.76(0.67$ to 0.87$)$ to 0.77 (0.68 to 0.88$)$ (data available on request).

There were statistically significant interaction effects of walking pace and total PA volume (eg, $p=0.038$ for ACM) and highest intensity reached (eg, $p=0.004$ for ACM). Significant interaction effects were also found for walking pace and age (eg, $\mathrm{p}=0.005$ for ACM) but not for sex or BMI.

Stratified analyses by age in two and three groups are presented in figure 1 and online supplementary figure 
Table 2 Associations between walking pace (three groups) and all-cause, cardiovascular disease and cancer mortality. Walkers aged 30 years and over with no diagnosed cardiovascular disease or cancer at baseline* excluding deaths occurring in the first 24 months of follow-up

\begin{tabular}{|c|c|c|c|c|c|c|c|}
\hline & \multirow[b]{2}{*}{ Deaths/n } & \multicolumn{2}{|c|}{ Model 1† } & \multicolumn{2}{|c|}{ Model 2‡ } & \multicolumn{2}{|c|}{ Model $3 \S$} \\
\hline & & HR & $95 \% \mathrm{Cls}$ & HR & $95 \% \mathrm{Cls}$ & HR & $95 \%$ Cls \\
\hline \multicolumn{8}{|l|}{ All-cause mortality } \\
\hline \multicolumn{8}{|l|}{ Walking pace } \\
\hline Slow & $576 / 4101$ & 1 & & 1 & & 1 & \\
\hline Average & $1957 / 25857$ & 0.73 & 0.67 to 0.81 & 0.78 & 0.71 to 0.87 & 0.80 & 0.72 to 0.88 \\
\hline Brisk/fast & 730/19 773 & 0.61 & 0.55 to 0.69 & 0.68 & 0.61 to 0.77 & 0.76 & 0.67 to 0.87 \\
\hline $\mathrm{P}$ trend linear & & $<0.001$ & & $<0.001$ & & $<0.001$ & \\
\hline $\mathrm{P}$ trend non-linear & & $<0.001$ & & 0.003 & & 0.001 & \\
\hline \multicolumn{8}{|c|}{ Cardiovascular mortality } \\
\hline \multicolumn{8}{|l|}{ Walking pace } \\
\hline Slow & $192 / 4101$ & 1 & & 1 & & 1 & \\
\hline Average & $552 / 25857$ & 0.68 & 0.57 to 0.81 & 0.75 & 0.63 to 0.90 & 0.76 & 0.64 to 0.91 \\
\hline Brisk/fast & $193 / 19773$ & 0.55 & 0.45 to 0.68 & 0.67 & 0.54 to 0.83 & 0.79 & 0.62 to 0.99 \\
\hline $\mathrm{P}$ trend linear & & $<0.001$ & & 0.001 & & 0.089 & \\
\hline$P$ trend non-linear & & 0.007 & & 0.032 & & 0.007 & \\
\hline \multicolumn{8}{|l|}{ Cancer mortality } \\
\hline \multicolumn{8}{|l|}{ Walking pace } \\
\hline Slow & $137 / 4101$ & 1 & & 1 & & 1 & \\
\hline Average & $717 / 25857$ & 1.03 & 0.85 to 1.24 & 1.06 & 0.88 to 1.29 & 1.08 & 0.89 to 1.31 \\
\hline Brisk/fast & 297/19 773 & 0.88 & 0.71 to 1.08 & 0.95 & 0.76 to 1.17 & 1.02 & 0.81 to 1.29 \\
\hline $\mathrm{P}$ trend linear & & 0.152 & & 0.478 & & 0.945 & \\
\hline $\mathrm{P}$ trend non-linear & & 0.338 & & 0.269 & & 0.339 & \\
\hline
\end{tabular}

The Health Survey for England and Scottish Health Survey ( $n=49731$ ).

*Prevalent cardiovascular disease was defined as doctor-diagnosed or self-reported (long-standing illness module) ischaemic heart disease, angina or stroke; prevalent cancer was determined through cancer registration records or self-reported (long-standing illness module).

†Model adjusted for age, sex and cohort.

¥Model also adjusted for long-standing illness, alcohol drinking frequency, psychological distress, body mass index, smoking status and education level.

$\S$ Model also adjusted for total (non-walking) physical activity volume (MET-hours/week), walking volume (MET-hours/week) and highest physical activity intensity reached.

MET, metabolic equivalent.

1 , respectively, and by compliance with the PA recommendations in figure 2. Figure 1 shows clearer evidence of a relationship between walking pace and all-cause and CVD mortality, but not cancer mortality, in the over 50s compared with the results for the whole sample. There was little evidence of association in the under $50 \mathrm{~s}$. Online supplementary figure 1 showed clearer evidence for a relationship of walking pace with ACM in those aged $45-59$ and $\geq 60$ years and with CVD mortality in those aged $\geq 60$ years.

Figure 2 shows clearer evidence of a relationship between walking pace and all-cause and CVD mortality, but not cancer mortality, among those that did not meet the PA guidelines compared with the results of the whole sample. For those meeting the guidelines, the direction of effect for all-cause and CVD mortality was protective for increasing pace, but very low number of events caused low power and wide CIs.

Figure 3 shows the stratified analyses of walking pace and all-cause and CVD mortality by highest intensity reached; analyses were not performed for cancer mortality due to the low number of events in some cells and the apparent violation of the proportional hazards assumption. There was evidence of a relationship between walking pace and ACM in both the light and moderate-intensity groups. There was some evidence for a relationship with CVD mortality in these groups although CIs were wider and there was no dose-response. There was no evidence of a relationship between walking pace and all-cause or CVD mortality among the group that reported reaching vigorous intensity.

\section{DISCUSSION}

In adults in Scotland and England, walking at average or brisk/ fast pace was associated with a reduced risk of all-cause and CVD mortality compared with walking at slow pace. However, there was no evidence of a similar relationship with cancer mortality. Our findings are in agreement with previous studies which have reported that a higher pace of walking was associated with a risk reduction for ACM between $19 \%{ }^{20}$ and $42 \% .^{11}$ Our estimates are within this range, and adjusted for total volume of both walking and non-walking PA (MET-hours/week), and highest PA intensity reached. We found that the associations between pace and ACM persisted after controlling for total leisure time PA, which is consistent with studies that controlled for total walking energy expenditure ${ }^{21}$ and MVPA. ${ }^{12}$ Batty et al reported a 20\% reduction in cancer mortality for walking fast. ${ }^{11}$ Similar to Yates et $a l,{ }^{12}$ we did not find any evidence of this effect.

\section{Possible explanations}

The association between pace on all-cause and CVD mortality may be explained by the increased relative exercise intensity elicited by a faster pace providing a greater stimulus for physiologic adaptations $^{22}$ in functions known to influence CVD mortality. This may be further confirmed by the observation that the associations of walking pace with ACM and (in particular) CVD mortality were considerably weakened for the subsample of participants who have achieved vigorous intensity in non-walking PA.

We did not find an effect of pace on cancer mortality. Volume may be more important than pace for cancer mortality. 
A All cause Mortality

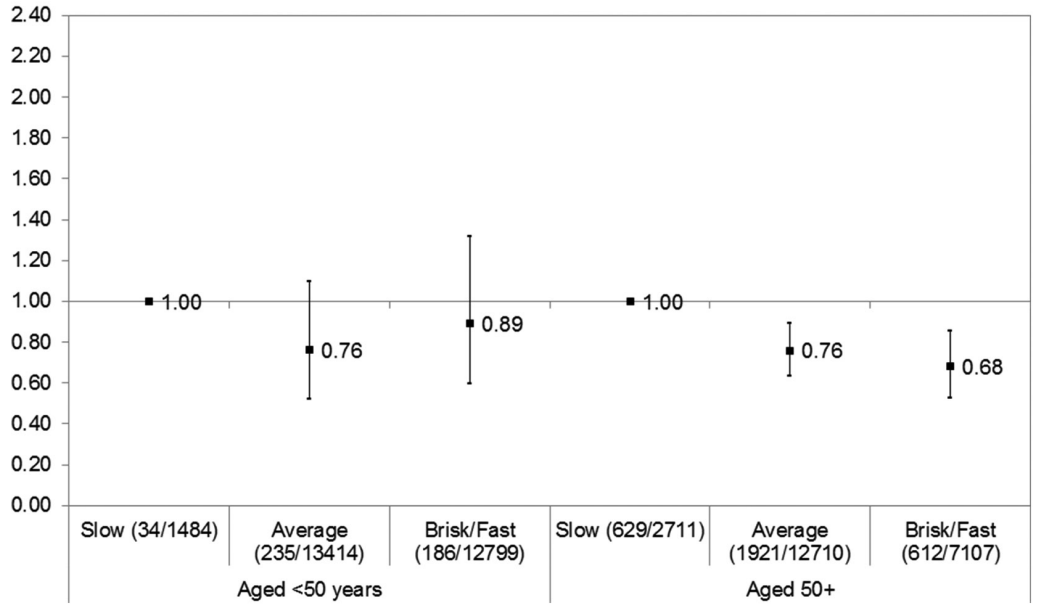

B CVD Mortality

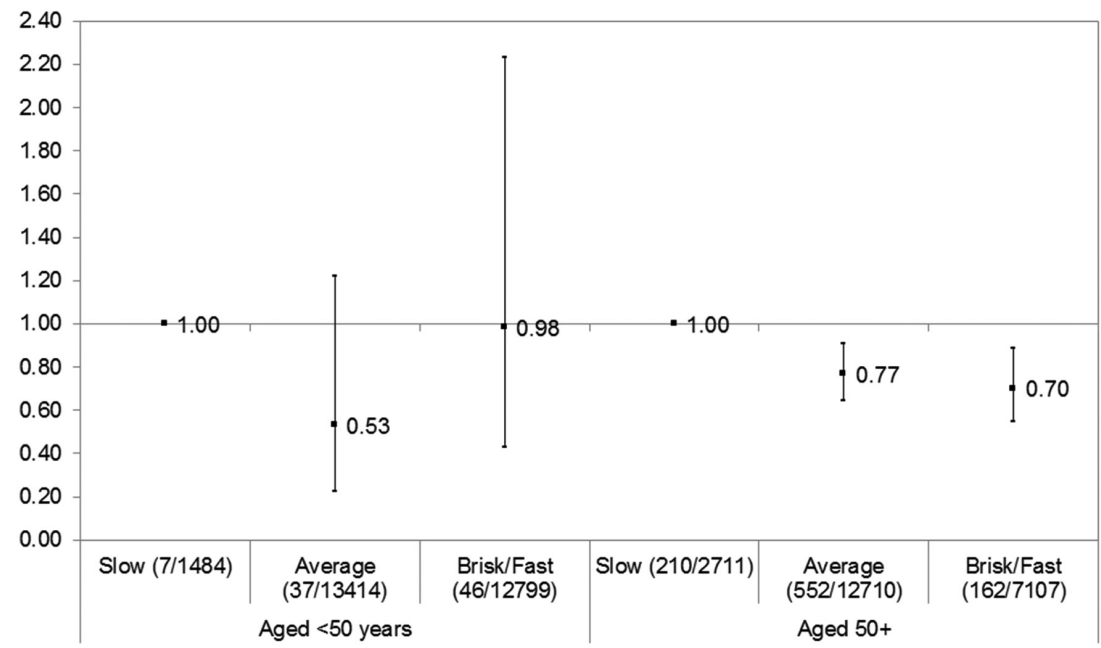

C Cancer Mortality

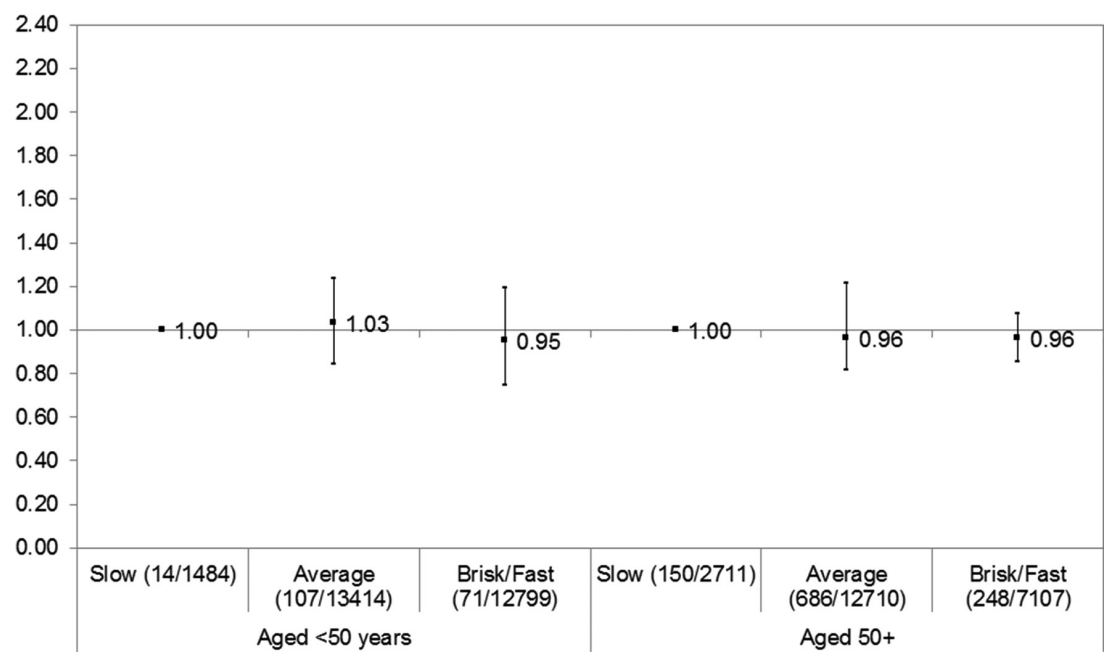

Figure 1 Associations between walking pace (three groups) and all-cause (A), cardiovascular disease (B) and cancer (C) mortality by age group ( $<50 \mathrm{vs} \geq 50$ years). Walkers aged 30 years and over with no diagnosed cardiovascular disease or cancer at baseline. The Health Survey for England and Scottish Health Survey. Fifty years of age was selected as a cut-off point due to its proximity to median age for this sample (48 years). (B) Prevalent cardiovascular disease was defined as doctor-diagnosed or self-reported (long-standing illness module) ischaemic heart disease, angina or stroke; prevalent cancer was determined through cancer registration records or self-reported (long-standing illness module). Model adjusted for sex, cohort, long-standing illness, alcohol drinking frequency, psychological distress, body mass index, smoking status, education level, total (non-walking) physical activity volume (MET-hours/week), walking volume (MET-hours/week) and highest physical activity intensity reached. CVD, cardiovascular disease; MET, metabolic equivalent. 
A All cause Mortality

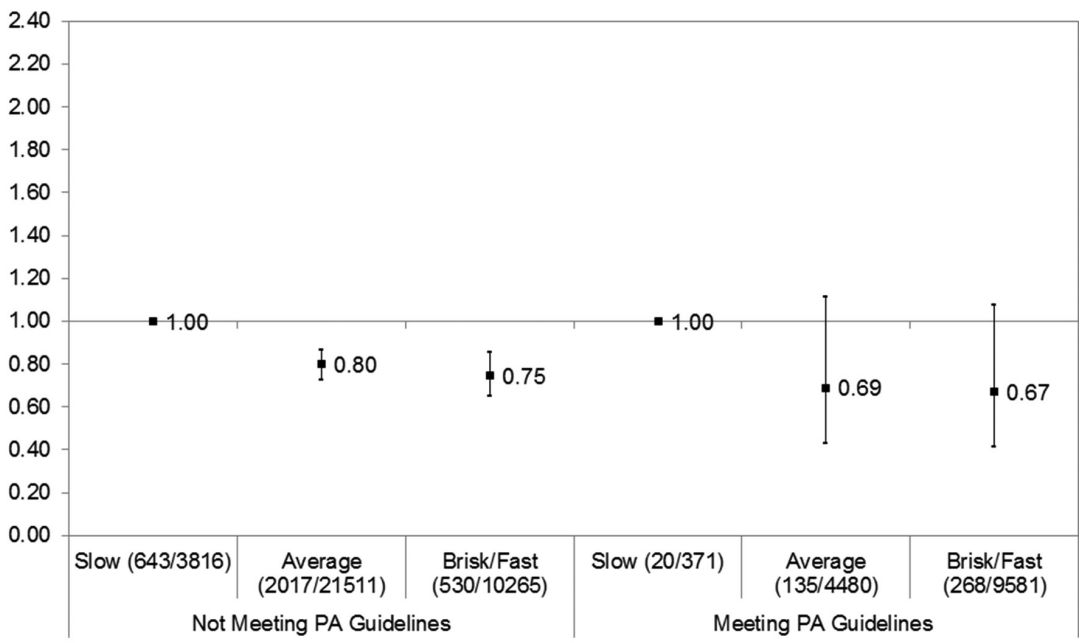

B CVD Mortality

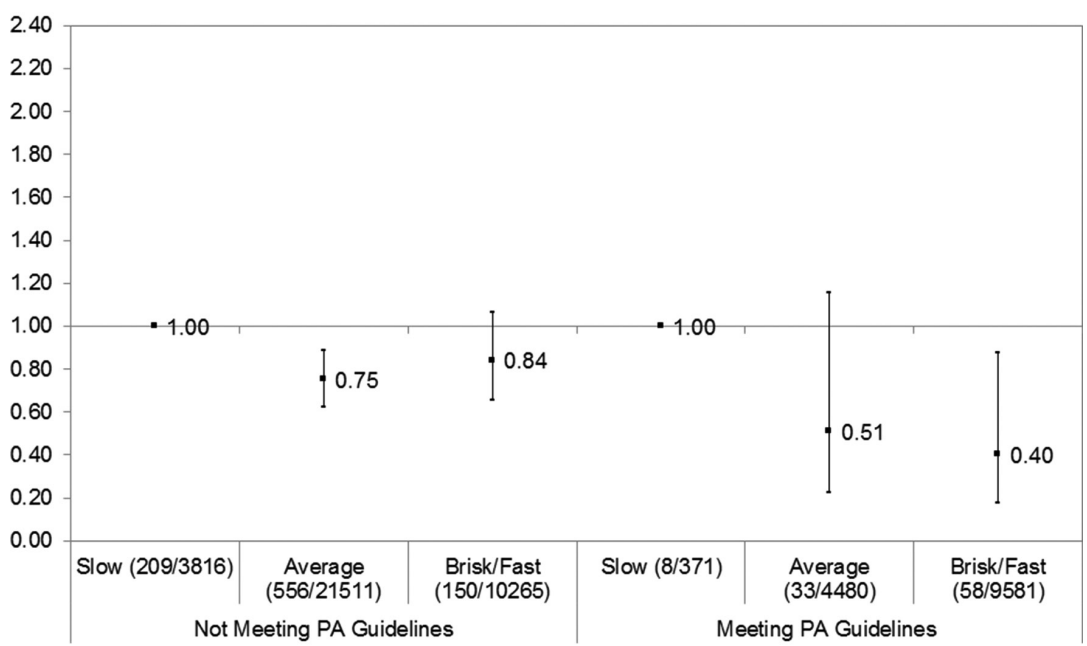

C Cancer Mortality

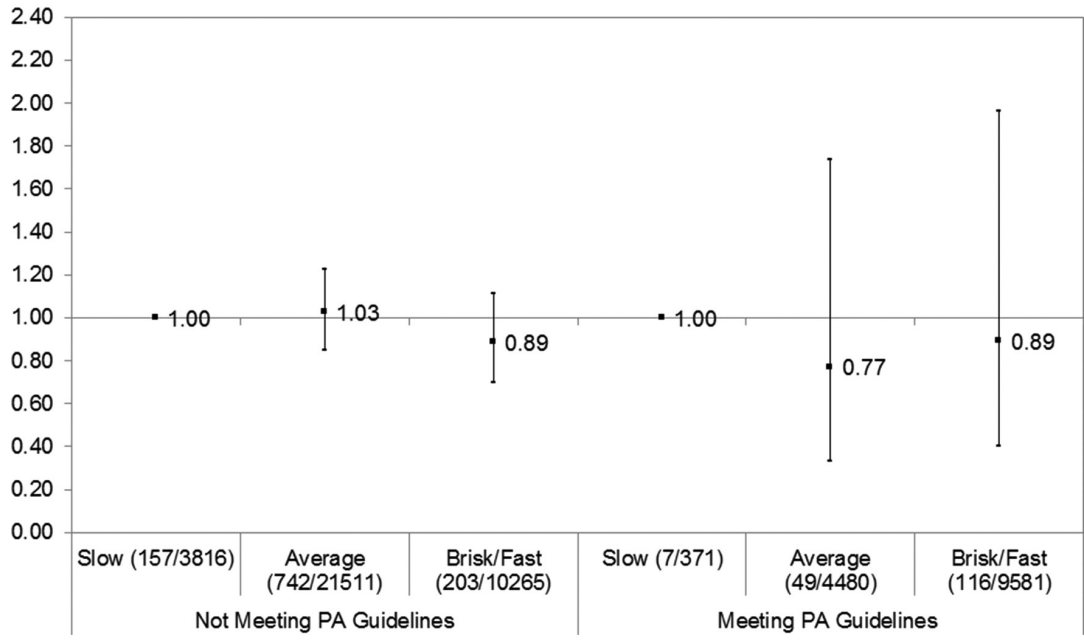

Figure 2 Associations between walking pace (three groups) and all-cause (A), cardiovascular disease (B) and cancer (C) mortality by physical activity level (meeting vs not meeting the physical activity recommendations). Walkers aged 30 years and over with no diagnosed cardiovascular disease or cancer at baseline. The Health Survey for England and Scottish Health Survey. Adherence to the physical activity recommendations was defined as at least $150 \mathrm{~min}$ of moderate-intensity activity or $75 \mathrm{~min} /$ week of vigorous-intensity activity or equivalent combinations of moderate and vigorous activities. Prevalent cardiovascular disease was defined as doctor-diagnosed or self-reported (long-standing illness module) ischaemic heart disease, angina or stroke; prevalent cancer was determined through cancer registration records or self-reported (long-standing illness module). Model adjusted for sex, cohort, long-standing illness, alcohol drinking frequency, psychological distress, body mass index, smoking status, education level, walking volume (MET-hours/week) and highest physical activity intensity reached. CVD, cardiovascular disease; MET, metabolic equivalent; PA, physical activity. 


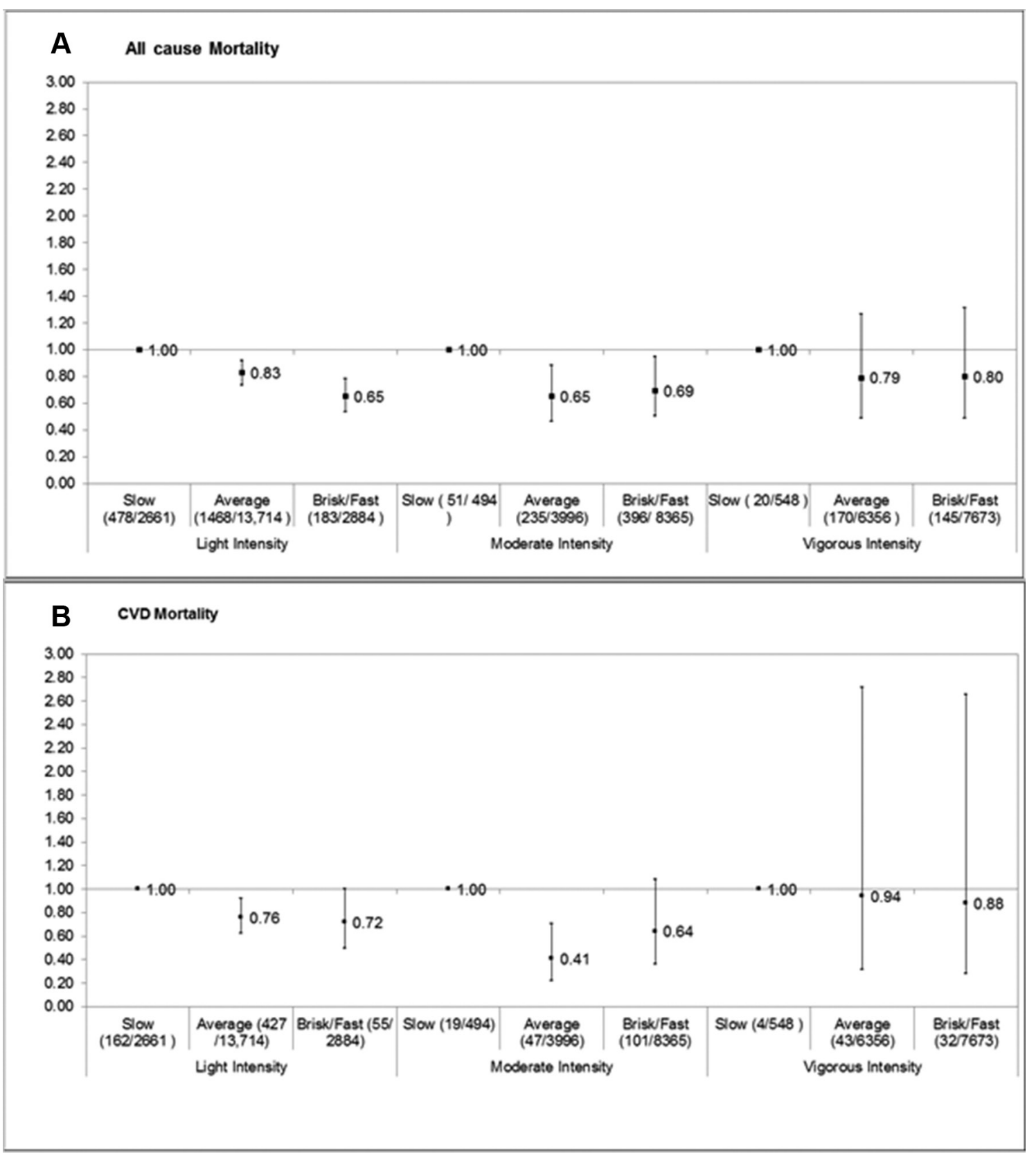

Figure 3 Associations between walking pace (three groups) and all-cause (A) and cardiovascular disease (B) mortality by highest physical activity intensity reached (light/moderate/vigorous). Walkers aged 30 years and over with no diagnosed cardiovascular disease or cancer at baseline. The Health Survey for England and Scottish Health Survey. Prevalent cardiovascular disease was defined as doctor-diagnosed or self-reported (longstanding illness module) ischaemic heart disease, angina or stroke; prevalent cancer was determined through cancer registration records or selfreported (long-standing illness module). Model adjusted for sex, cohort, long-standing illness, alcohol drinking frequency, psychological distress, body mass index, smoking status, education level, walking volume (MET-hours/week) and total (non-walking) physical activity volume (MET-hours/ week). CVD, cardiovascular disease; MET, metabolic equivalent.

Alternatively, we know that different cancers have different relationships with PA, and that if we had examined mortality from specific malignancies, for example, breast and colon cancers, a relationship may have been observed. ${ }^{23}$

We did not find evidence for associations for the younger participants, the physically active or for those reaching vigorous intensity, but recommend caution when interpreting these findings as low number of events in some strata increased uncertainty. It is possible that older age and lower PA status (total or intensity) predict lower aerobic fitness (maximal oxygen consumption). As such, that the relative intensity of walking at faster pace may be equivalent to the upper end of moderate intensity or even vigorous intensity, and therefore provides a greater physiological stimulus for maintaining cardiovascular function and promoting health.

Separating the effect of one specific aspect of PA and understanding its potentially causal association with mortality is complex. Our analyses suggest that participants who usually walk at a brisk/fast pace are overall the most active and probably the healthiest. Although it is biologically plausible that walking at a higher pace leads to better health overall and cardiovascular health specifically, it is also likely that walking at a faster pace is a marker for better health, fitness and physical function, which predicts the risk for mortality in 
the following years. In other words, walking pace may be a predictor of lower mortality risk, a causal factor, or both.

\section{Strengths and limitations}

The strengths of the present study include the large sample comprising a series of baseline surveys that were roughly representative of the population in England and Scotland, the very high response rates and the relatively long follow-up. The results can be generalised to the UK population with more confidence than previous estimates. To our knowledge, this is the first such study to report associations between walking pace and all-cause, CVD and cancer mortality and adjust for total (walking and non-walking) PA volume and highest intensity reached. We also present novel analysis of associations stratified by age, total PA and highest intensity reached to investigate important potential effect modifiers.

Limitations include the exposure 'walking pace' and all other PA variables were self-reported and therefore subject to misclassification and other biases. Further misclassification may have been introduced by the imputation of walking duration for a number of baseline surveys, ${ }^{16}$ and this may be partly the reason why adjustments for total walking volume had negligible impact on the estimates. The repeated cross-sectional nature of HSE and SHeS meant we could not assess or account for temporal changes in walking behaviour within individuals. The analyses controlled for a comprehensive set of covariates in addition to PA, although we cannot discount the possibility of residual confounding. Some stratified analyses had too few events and therefore may not have been powerful enough to detect associations or lack of association with confidence.

\section{Implications and future research}

The additional protective effect demonstrated from higher walking pace may have implications for public health messaging. Walking is a cornerstone of PA promotion for public health, but volume of walking (steps per day) has often been emphasised. ${ }^{24}$ Given the perceived time barrier cited by those who fail to meet current PA guidelines, a pace change may be more feasible (for those with adequate physical capacity) than increased volume or duration. We encourage the Chief Medical Officers' Physical Activity Guidelines Committee to consider this in their upcoming revision of the PA Guidelines. Further experimental research is warranted to establish if a randomised intervention based on pace elicits important physiological change. ${ }^{25}$

\section{CONCLUSIONS}

Walking is known to benefit health. Assuming causal relationships, these analyses suggest that increasing walking pace could be linked with lower risk for all-cause and CVD mortality. Walking pace should be emphasised in public health messages, especially in circumstances when increase in walking volume or frequency is less feasible.

Funding This analysis was not financially supported directly by any individual, agency or institution. The harmonisation of the pooled data sets used in this analysis was funded by the National Institute for Health Research (UK) through a grant to ES. ES and DD are funded by the National Health and Medical Research Council (Australia) through a Senior Research Fellowship and an Early Career Research Fellowship, respectively.

Competing interests None declared.

Patient consent Obtained.

Ethics approval Multiple Research Ethics Committees in England and Scotland.
Provenance and peer review Not commissioned; externally peer reviewed.

(C) Article author(s) (or their employer(s) unless otherwise stated in the text of the article) 2018. All rights reserved. No commercial use is permitted unless otherwise expressly granted.

\section{REFERENCES}

1 Kelly P, Murphy M, Mutrie N. The health benefits of walking. walking: connecting sustainable transport with health: Emerald Publishing Limited, 2017:61-79.

2 Hamer M, Chida Y. Walking and primary prevention: a meta-analysis of prospective cohort studies. Br J Sports Med 2008;42:238-43.

3 Woodcock J, Franco OH, Orsini N, et al. Non-vigorous physical activity and all-cause mortality: systematic review and meta-analysis of cohort studies. Int J Epidemiol 2011;40:121-38

4 Samitz G, Egger M, Zwahlen M. Domains of physical activity and all-cause mortality: systematic review and dose-response meta-analysis of cohort studies. Int J Epidemiol 2011:40:1382-400.

5 Kelly P, Kahlmeier S, Götschi T, et al. Systematic review and meta-analysis of reduction in all-cause mortality from walking and cycling and shape of dose response relationship. Int J Behav Nutr Phys Act 2014;11:132.

6 Office for National Statistics (UK). Avoidable mortality in England and Wales: 2015. 2017 https://www.ons.gov.uk/peoplepopulationandcommunity/healthandsocialcare/c ausesofdeath/bulletins/avoidablemortalityinenglandandwales/2015 (accessed 19 Jan 2018).

7 Celis-Morales CA, Lyall DM, Welsh P, et al. Association between active commuting and incident cardiovascular disease, cancer, and mortality: prospective cohort study. BMJ 2017;357:j1456.

8 Matthews CE, Jurj AL, Shu XO, et al. Influence of exercise, walking, cycling, and overall nonexercise physical activity on mortality in Chinese women. Am J Epidemiol 2007:165:1343-50.

9 Saevereid HA, Schnohr P, Prescott E. Speed and duration of walking and other leisure time physical activity and the risk of heart failure: a prospective cohort study from the Copenhagen City Heart Study. PLoS One 2014;9:e89909.

10 Manson JE, Greenland P, LaCroix AZ, et al. Walking compared with vigorous exercise for the prevention of cardiovascular events in women. $N$ Engl J Med 2002;347:716-25.

11 Batty GD, Shipley MJ, Kivimaki M, et al. Walking pace, leisure time physical activity, and resting heart rate in relation to disease-specific mortality in London: 40 years follow-up of the original Whitehall study. An update of our work with professor Jerry N. Morris (1910-2009). Ann Epidemiol 2010;20:661-9.

12 Yates T, Zaccardi F, Dhalwani NN, et al. Association of walking pace and handgrip strength with all-cause, cardiovascular, and cancer mortality: a UK Biobank observational study. Eur Heart J 2017;38:3232-40.

13 Ebrahim S, Davey Smith G. Commentary: should we always deliberately be nonrepresentative? Int J Epidemiol 2013;42:1022-6.

14 Mindell J, Biddulph JP, Hirani V, et al. Cohort profile: the health survey for England. Int J Epidemiol 2012;41:1585-93.

15 Gray L, Batty GD, Craig P, et al. Cohort profile: the Scottish health surveys cohort: linkage of study participants to routinely collected records for mortality, hospital discharge, cancer and offspring birth characteristics in three nationwide studies. Int J Epidemiol 2010;39:345-50.

16 Stamatakis E, Ekelund U, Wareham NJ. Temporal trends in physical activity in England: The Health Survey for England 1991 to 2004. Prev Med 2007;45:416-23.

17 Scholes S, Coombs N, Pedisic Z, et al. Age- and sex-specific criterion validity of the health survey for England physical activity and sedentary behavior assessment questionnaire as compared with accelerometry. Am J Epidemiol 2014;179:1493-502.

18 Ainsworth BE, Haskell WL, Herrmann SD, et al. 2011 Compendium of physical activities: a second update of codes and MET values. Med Sci Sports Exerc 2011;43:1575-81.

19 World Health Organization. Global recommendations on physical activity for health Geneva:WHO, 2010.

20 Stanaway FF, Gnjidic D, Blyth FM, et al. How fast does the Grim Reaper walk? Receiver operating characteristics curve analysis in healthy men aged 70 and over. BMJ 2011;343:d7679.

21 Williams PT, Thompson PD. The relationship of walking intensity to total and cause-specific mortality. results from the national walkers' health study. PLoS One 2013:8:e81098.

22 McArdle WD, Katch Fl, Katch VL, et al. Exercise physiology. energy, nutrition and human performance. 5th edn. Baltimore: Lippincott Williams \& Wilkins, 2001.

23 McTiernan A. Mechanisms linking physical activity with cancer. Nat Rev Cancer 2008;8:205-11.

24 European Commission. Special eurobarometer 412. sport and physical activity. Brussels: DG COMM "Strategy, Corporate Communication Actions and Eurobarometer" Unit, 2015.

25 Duncan JJ. Women walking for health and fitness. How much is enough? JAMA 1991;266:3295-9. 